\title{
SIRT1 overexpression in cervical squamous intraepithelial lesions and invasive squamous cell carcinoma
}

\section{Summary:}

Invasive squamous cell carcinoma (SCC) of the cervix involves the progression of premalignant cervical intraepithelial neoplasia (CIN) and is associated with persistent human papilloma virus (HPV) infection. Most CINs will regress, and the challenge is to identify the lesions likely to progress to invasive cancer. We evaluated Sirtuin1 (SIRT1) expression in non-neoplastic cervix, CINs and SCC as a potential biomarker to predict disease progression. A total of 101 cases were selected including 29 CIN 1, 32 CIN 2, 16 CIN 3, 2 microinvasive SCC and 22 invasive SCC. Cervical non-neoplastic squamous epithelium showed weak positivity of SIRT1 in the basal layer. SIRT1 cytoplasmic over-expression was found in $13.8 \%$ CIN 1 (4/29), 40.6\% CIN2 (13/32), and 50\% CIN3 (8/16), and it was statistically significant between CIN1 and CIN2/3 lesions $(p=0.01)$. All 24 cases of invasive and microinvasive SCC showed SIRT1 overexpression with $25 \%(6 / 24)$ showing cytoplasmic staining only, $4.2 \%(1 / 24)$ showing nuclear staining only, and $70.8 \%$ (17/24) showing both nuclear and cytoplasmic staining. From CIN 1 to SCC, SIRT1 expression showed steady and statistically significant increase (CIN1 vs CIN2-3, $p=0.01$; CIN2-3 vs SCC, $p=0.0001$ ). Thus, SIRT1 may serve as a potential biomarker for predicting the progression of CIN to invasive SCC.

\section{Keywords:}

Sirtuin1 (SIRT1), cervical squamous neoplasia (CIN), squamous cell carcinoma (SCC)

\section{Introduction:}

The incidence rates of invasive cervical cancer have markedly decreased in the past decades due to the introduction of cervical screening program for cervical intraepithelial neoplasia (CIN) and the therapeutic excision of premalignant CIN lesions. Nonetheless, approximately 12,900 new cases and 4,100 estimated deaths were expected in 2015 [1]. The development of invasive squamous cell carcinoma (SCC) of the uterine cervix involves the progression of premalignant lesions and is associated with persistent infection of high-risk Human Papilloma Viruses (HPV),

such as HPV 16 and 18. A key event in disease progression is related to the upregulation of viral proto-oncogenes E6 and E7, which inhibits p53 and retinoblastoma tumor suppression protein 
(pRb) respectively, halts apoptosis, and abolishes cell-cycle arrest [2-3]. On the other hand, biomarkers that can predict malignant progression of CINs are not well established and therefore, represent an area of great clinical interest.

Sirtuins (SIRT) are the mammalian homolog of Sir2 (silent information regulator 2) family of class III histone deacetylases dependent on nicotinamide adenine dinucleotide (NAD+) [4-5]. There are 7 isoforms producing SIRT1-7 found in different subcellular locations, such as cytoplasm, nucleus, and mitochondria [5-8]. SIRT1, a primarily nuclear deacetylase, may be expressed in variable subcellular localization depending on tissue type, and may undergo nucleocytoplasmic shuttling [9]. Its deacetylase activities can target histones and non-histones proteins, regulating pathways involved in cell proliferation, apoptosis, senescence, metabolism, and stress response, among other functions [6]. The target proteins include p53, fork-head class O transcription factor (FOXO), pRb, Ku 70, mismatch repair gene MLH1 and survivin, among others [10-11].

The role of SIRT1 in cancerogenesis is not clear and has been controversial with evidence supporting both tumor promoting and tumor suppressing functions depending on the specific tissue and distribution of factors [6,11-13]. SIRT1 has been shown to inhibit proliferation and tumor formation of colon cancer, and has been associated with good prognosis in squamous cell carcinomas of the head and neck [14-15]. On the other hand, SIRT1 overexpression has also been associated with tumor progression and unfavorable prognosis in breast, prostate, lung, gastrointestinal cancers (i.e. hepatocellular carcinoma, gastric cancer), and lymphoma [6,16-22]. In gynecological tumors, elevated expression of SIRT1 was seen more frequently in malignant ovarian epithelial tumors compared to benign lesions, and was associated with chemotherapy 
resistance in endometrial carcinoma cell lines and lower efficacy of neoadjuvant chemotherapy in locally advanced uterine cervical cancer [23-25]. Also, a study performed by Allison and colleagues demonstrated that HPV16 E7 unregulated SIRT1 levels in human cervical cancer cell lines [26]. However, the role of SIRT1 in uterine cervical CINs and SCC has not been well investigated.

In the study herein, we evaluated the expression of SIRT1 using immunohistochemistry in normal cervical squamous epithelium, CIN 1-3, and invasive SCC. Our goal was to study the potential value of SIRT1 as a biomarker for predicting malignant progression of cervical squamous intraepithelial neoplasms.

\section{Materials and Methods:}

\subsection{Tissue specimens}

A total of 101 tissue specimens from the uterine cervix were selected and evaluated in two phases. Phase I study involved archived material from Louisiana State University Health Science Center in Shreveport, Louisiana. A total of 77 cases were selected including 29 CIN 1, 32 CIN 2, and 16 CIN 3 from 58 cervical biopsies and 19 loop electrosurgical excision procedure (LEEP) specimens. Phase II study involved archived material from the University of Texas Health Science Center at Houston. A total of 24 cases were selected including 22 cases of uterine cervical invasive SCC and 2 cases of microinvasive SCC from 15 cervical biopsies, 5 LEEP or cervical conization specimens, and 4 from hysterectomy specimens. Uninvolved cervical epithelium from all specimens was used as the baseline expression of SIRT1. All cases were retrospectively reviewed and the diagnosis was confirmed. Phase I and phase II studies were approved by the Institutional Research Board for the Protection of Human Subjects of Louisiana 
State University Health Science Center at Shreveport, LA, and the University of Texas Health Science Center at Houston, TX, respectively.

\subsection{Immunohistochemical analysis}

Both SIRT1 and p16INK4a were examined in all CINs and only SIRT1 expression was evaluated in invasive SCC. Immunohistochemistry was performed using formalin-fixed paraffin-embedded tissue sectioned into $4-5 \mu \mathrm{m}$ sections with adequate positive and negative controls. Tissue slides were deparaffinized in xylene, rehydrated in graded alcohol, and heat treated in a steamer for $45 \mathrm{~min}$ in an Antigen Retrieval solution. After cooled down in room temperature in antigen retrieval solution, slides were then treated with $3 \% \mathrm{H}_{2} \mathrm{O}_{2}$ for 10 min to quench endogenous peroxidase activity, and blocked with horse serum (2.5\%) for $20 \mathrm{~min}$. Immunohistochemistry was manually performed using Shandon Coverplate Technology (Thermo Shandon Lt), by incubating the slides with $100 \mu 1$ the primary antibody (SIRT1, 1:100, Abcam; p16, 1:25, DAKO) for 1 hour at room temperature, 3 times washing with 1x PBS, then applying ABC Ready-To-Use reagents (R.T.U. VECTASTAIN, ABC kit, Vector Laboratories) at room temperature, according to manufactory instructions. EnVision FLEX DAB chromogen system (DAKO) was used for color development and the time ranged from 3-8 minutes depending on the color intensity. Positive and negative controls were included in each run. Mayer's Hematoxylin stain was used as the counterstain, and dehydration was performed using graded alcohol and xylene. All slides were reviewed using light microscopy in a double-blinded fashion. Each slide was scored depending on their staining intensity (0-3+) as: no staining (0), weak (1+), moderate (2+) and strong (3+). Cases with a score of $0-1+$ were considered negative, while those with a score of $2-3+$ were considered positive. Those cases showing positivity in 
$\leq 50 \%$ of tumor were considered as focal positive, while those with $>50 \%$ positive stain were considered as diffuse positive.

\subsection{Statistical analysis}

Fisher's exact test was used for the statistical analysis and $p$ value of $\leq 0.05$ was considered as statistically significant.

\section{Results:}

The uninvolved cervical squamous epithelium showed negative or weak SIRT1 positivity in the basal layer (Figure 1A-1B). In CIN lesions, dysplastic squamous cells showed cytoplasmic positivity for SIRT1 in an increasing fashion from low grade to high grade intraepithelial neoplasm. In CIN 1, 4 out of 29 cases (13.8\%) were positive for SIRT1 (Figure 1C-1D). In high grade lesions, 13 out of 32 cases (40.6\%) of CIN 2 (Figure 2A-2B) and 8 out of 16 cases (50\%)

of CIN 3 (Figure 2C-2D) showed increased expression of SIRT1. The SIRT1 expression between CIN 1 and CIN 2-3 was significantly increased (4/29 vs 21/48, $p=0.01$ ). All 24 cases of SCC (including 2 microinvasive SCC) showed strong SIRT1 expression (2-3+) in neoplastic cells with 17 out of 22 cases (68\%) showing both nuclear and cytoplasmic positivity, 6 out of 24 (25\%) showing cytoplasmic staining only, and 1 out of 22 cases (4\%) showing nuclear staining only (Figure 3A-3D). Two microinvasive SCC showed cytoplasmic and nuclear overexpression of SIRT1. From CIN 2-3 to invasive SCC, SIRT1 expression also showed a statistically significant increase ( $21 / 48$ vs $24 / 24, p=0.0001)$. In our study, nuclear SIRT1 positivity was only observed in invasive SCC. These findings are summarized in Table 1. 
Strong and diffuse p16INK4a positivity was observed in 6 out of 29 cases (20.6\%) of CIN 1, in 26 out of 32 cases $(81.2 \%)$ of CIN 2, and in all the cases (16/16) of CIN 3 (Figure 4A-4B). The p16INK4a expression was statistically significant between the CIN 1 and CIN 2-3 (6/29 vs 42/48, $p=0.0001)$.

\section{Discussion:}

Our results show that increased expression of SIRT1 correlates with disease progression of CINs. SIRT1 overexpression was observed in $13.8 \%$ (4/29) of CIN 1, 40.6\% (13/32) CIN 2, 50\% (8/16) CIN 3, and 100\% (24/24) of microinvasive and invasive SCC. There is only one reported study in the literature about SIRT1 expression in CINs. Singh and colleagues found a significant increase in SIRT1 expression in CINs when compared to non-neoplastic tissue [27]. Their study also noted the nuclear expression of SIRT1 in CINs and SCC. However, their results did not

yield a significant difference in SIRT1 expression between low-grade and high-grade CINs, and between SCC and CINs. This discrepancy between our study and their results may be related to the scoring method and the different SIRT1 antibodies used in these 2 studies. The discrepancy needs further studies for clarification.

Additionally, our findings showed nuclear expression of SIRT1 in a number of invasive carcinomas but not in CIN lesions. This shift in expression may be explained by a nucleocytoplasmic shuttling mechanism previously described by Tanno, et al [9]. The significance of SIRT1 nuclear expression in SCC is not clear. However, the nuclear expression was only noted in invasive SCC, which may be associated with other genetic abnormality during the disease progression. 
The biological explanation for the upregulation of cellular SIRT1 in CINs and SCC may be related to the viral oncoprotein HPV E7. Studies have shown that selective silencing of HPV E7 using RNA interference in SiHa cervical cancer cells results in SIRT1 down-regulation, loss of hyper-pRb proteins, and induction of apoptotic cell death [26,28-30]. In contrast, HPV E6 silencing induces accumulation of cellular p53 and p21 proteins, and cell growth arrest [26,2830]. SIRT1 overexpression by HPV E7 results in cell death prevention and p53 deacetylation hindering its pro-apoptotic functions $[10,26]$. Based on these findings, Allison et al. proposed a new model for malignant cell transformation of cervical epithelial cells in which HPV viral oncoproteins E6 and E7 target cellular p53 and SIRT1 respectively and disrupt homeostatic regulatory mechanisms [26]. The exact mechanism by which HPV E7 increases SIRT1 expression is not yet elucidated, but they suggested that it may take place at the posttranscriptional level [26].

Understanding the role of SIRT1 in cervical cancer tumorigenesis may have both prognostic and therapeutic value. One study showed that high SIRT1 expression in locally advanced cervical cancer was associated with poor prognosis and poor therapy response [24]. Although most low grade CIN lesions regress, few cases progress to invasive carcinoma. The challenge is to identify the lesions that have a higher tendency of malignant transformation and provide treatment while preventing overtreatment of the low risk lesions. Some biomarkers have shown correlation with the progression of CINs, including HPV genotype (such as HPV 16, 18 and 45), p16, and Ki-67. Our study showed that SIRT1 overexpression is a potential biomarker for predicting CIN progression.

SIRT1 could also be a therapeutic target using SIRT1 inhibitors and known inhibitors of sirtuins, including splitomicin, sirtinol, AGK2, cambinol, indol derivatives (EX-527), and tenovin, among 
others [5]. Singh et al. evaluated the effect of Sirtinol, Ex527 and AGK2 in cervical cancer cells [27]. They reported induced cell death when tumor cells were treated with Sirtinol, a general sirtuin inhibitor, and AGK2, a SIRT2 inhibitor. Other studies using breast and endometrial carcinoma cells lines have also reported significant growth suppression with Ex527, a SIRT1 inhibitor [25, 31].

Our study observed differences of SIRT1 overexpression among low grade CIN, high grade CIN and invasive carcinoma. Our findings may correlate with the genetic abnormalities during disease progression. One systemic review and meta-analytical assessment of chromosomal aberrancies in HPV-associated neoplasia revealed that the most common alteration in HPV-16 cervical SCC were gains at chromosome $3 \mathrm{q}$, which were also identified in more than $25 \%$ of high-grade CIN; and that chromosomal abnormalities in low grade CIN were rare [32]. Moreover, array-based comparative genomic hybridization of cervical neoplasms demonstrated that a subset of CIN 2-3 had similar chromosomal profiles of SCC, including gains of chromosome 1q, 3q and 20q [33]. Amplification of chromosome 20q has been associated with expression of HPV-16 E7 and chromosome instability at high levels in uroepithelial, airway and anogenital epithelial cells [34-35]. These results suggest that specific genomic alterations occurring in pre-neoplastic squamous lesions of the cervix have a higher tendency of malignant transformation, and those genetic changes are corresponding and similar to our observation of SIRT1 expression in CINs and invasive SCC. Further studies to compare genetic alterations and SIRT1 overexpression may clarify this link.

\section{Concluding Remarks}


In summary, our study showed that SIRT1 overexpression is present in cervical SCC and in a large proportion of CIN 2-3, but is rare in CIN 1. SIRT1 expression showed steady and statistically significant increase from non-neoplastic tissue to CIN 2-3, and to invasive SCC. Therefore, SIRT1 overexpression may be associated with disease progression, and therefore may be used as a prognostic biomarker. Further prospective studies are needed to confirm the potential role of SIRT1 as a biomarker for malignant transformation of cervical pre-neoplastic lesions. 


\section{References:}

[1] Siegel RL, Miller KD, Jemal A. Cancer statistics, 2015. CA Cancer J Clin. 2015;65(1):5-29. [2] Schiffman M, Castle PE, Rodriguez AC, et al. Human papillomavirus and cervical cancer. Lancet. 2007;370:890-907.

[3] von Knebel Doeberitz M, Vinokurova S. Host factors in HPV-related carcinogenesis: cellular mechanisms controlling HPV infections. Arch Med Res. 2009;40(6):435-42.

[4] Landry J, Sutton A, Tafrov ST, et al. The silencing protein SIR2 and its homologs are NADdependent protein deacetylases. Proc Natl Acad Sci U S A. 2000;97(11):5807-11.

[5] Villalba JM, Alcain FJ. Sirtuin activators and inhibitors. Biofactors. 2012;38(5):349-59.

[6] Yuan H, Su L, Chen WY. The emerging and diverse roles of sirtuins in cancer: a clinical perspective. Onco Targets Ther. 2013;6:1399-416.

[7] Frye RA. Characterization of five human cDNAs with homology to the yeast SIR2 gene: Sir2-like proteins (sirtuins) metabolize NAD and may have protein ADP-ribosyltransferase activity. Biochem Biophys Res Commun. 1999;260:273-9.

[8] Frye RA. Phylogenetic classification of prokaryotic and eukaryotic Sir2-like proteins. Biochem Biophys Res Commun. 2000;273:793-8.

[9] Tanno M, Sakamoto J, Miura T, et al. Nucleocytoplasmic shuttling of the NAD ${ }^{+}$-dependent histone deacetylase SIRT1. J Biol Chem. 2007;282(9):6823-32.

[10] Brooks CL, Gu W. Anti-aging protein SIRT1: a role in cervical cancer? Aging. 2009;1(3):278-80.

[11] Deng CX. SIRT1, is it a tumor promoter or tumor suppressor? Int J Biol Sci. 2009;5:147-52. [12] Song NY, Surh YJ. Janus-faced role of SIRT1 in tumorigenesis. Ann NY Acad Sci. 2012;1271(1):10-9.

[13] Bosch-Presegué L, Vaquero A. The dual role of sirtuins in cancer. Genes Cancer. 2011;2(6):648-62.

[14] Kabra N, Li Z, Chen L, et al. SIRT1 is an inhibitor of proliferation and tumor formation in colon cancer. J Biol Chem 2009;284:18210-7.

[15] Nogushi A, Li X, Kubota A, et al. SIRT1 expression is associated with good prognosis for head and neck squamous cell carcinoma patients. Oral Surg Oral Med Oral Pathol Oral Radiol. 2013;115(3):385-92.

[16] Lee H, Kim KR, Noh SJ, et al. Expression of DBC1 and SIRT1 is associated with poor prognosis for breast carcinoma. Hum Pathol. 2011;42(2):204-13.

[17] Byles V, Zhu L, Lovaas JD, et al. SIRT1 induces EMT by cooperating with EMT transcription factors and enhances prostate cancer cell migration and metastasis. Oncogene. 2012;31(43):4619-29.

[18] Noh SJ, Baek HA, Park HS, et al. Expression of SIRT1 and cortactin is associated with progression of non-small cell lung cancer. Pathol Res Pract. 2013;209(6):365-70.

[19] Kriegl L, Vieth M, Kirchner T, et al. Up-regulation of c-MYC and SIRT1 expression correlates with malignant transformation in the serrated route to colorectal cancer. Oncotarget. 2012;3(10):1182-93. 
[20] Cha EJ, Noh SJ, Kown KS, et al. Expression of DBC1 and SIRT1 is associated with poor prognosis of gastric carcinoma. Clin Cancer Res. 2009;15(13):4453-9.

[21] Chen HC, Jeng YM, Yuan RH, et al. SIRT1 promotes tumorigenesis and resistance to chemotherapy in hepatocellular carcinoma and its expression predicts poor prognosis. Ann Surg Oncol. 2012;19(6):2011-9.

[22] Jang KY, Hwang SH, Kwon KS, et al. SIRT1 expression is associated with poor prognosis of diffuse large B-cell lymphoma. Am J Surg Pathol. 2008;32(10):1523-31.

[23] Jang KY, Kim HS, Hwang SH, et al. Expression and prognostic significance of SIRT1 in ovarian epithelial tumours. Pathology. 2009;41(4):366-71.

[24] Teramae M, Fukuda T, Wada T, et al. Sirtuin1 expression predicts the efficacy of neoadjuvant chemotherapy for locally advances uterine cervical cancer. Mol Clin Oncol. 2015;3(1):73-8.

[25] Asaka R, Miyamoto T, Yamada Y, et al. Sirtuin 1 promotes the growth and cisplatin resistance of endometrial carcinoma cells: a novel therapeutic target. Lab Invest.

2015;95(12):1363-73.

[26] Allison SJ, Jiang M, Milner J. Oncogenic viral protein HPV E7 up-regulates the SIRT1 longevity protein in human cervical cancer cells. Aging. 2009;1(3):316-27.

[27] Singh S, Kumar U, Thakur S, et al. Expression/localization patterns of sirtuins (SIRT1, SIRT2, and SIRT7) during progression of cervical cancer and effects of sirtuin inhibitors on growth of cervical cancer cells. Tumor Biol. 2015;36(8):6159-71.

[28] Jiang M, Milner J. Selective silencing of viral gene expression in HPV-positive human cervical carcinoma cells treated with siRNA, a primer of RNA interference. Oncogene. 2002;21(39):6041-8.

[29] Jiang M, Milner J. Selective silencing of viral gene E6 and E7 expression in HPV-positive human cervical carcinoma cells using small interfering RNAs. Method Mol Biol. 2005;292:40120.

[30] Sima N, Wang W, Kong D, et al. RNA interference against HPV16 E7 oncogene leads to viral E6 and E7 suppression in cervical cancer cells and apoptosis via upregulation of Rb and p53. Apoptosis. 2008;13(2):273-81.

[31] Peck B, Chen CY, Ho KK, et al. SIRT1 inhibitors induce cell death and p53 acetylation through targeting both SIRT1 and SIRT2. Mol Cancer Ther. 2010;9(4):844-55.

[32] Thomas LK, Bermejo JL, Vinokurova S, et al. Chromosomal gains and losses in human papillomavirus-associated neoplasia of the lower genital tract- a systemic review and metaanalysis. Eur J Cancer. 2014;50(1):85-98.

[33] Wilting SM, Steenbergen RDM, Tijseen M, et al. Chromosomal signatures of a subset of high-grade premalignant cervical lesions closely resemble invasive carcinomas. Cancer Res. 2009;69(2):647-55.

[34] Savelieva E, Belair CD, Newton MA, et al. 20q gain associates with immortalization: 20q13.2 amplification correlates with genome instability in human papillomavirus $16 \mathrm{E} 7$ transformed human uroepithelial cells. Oncogene. 1997;14(5):551-60.

[35] Klingelhutz AJ, Qian Q, Phillips SL, et al. Amplification of the chromosome 20q region is associated with expression of HPV-16 E7 in human airway and anogenital epithelial cells. Virology. 2005;340(2):237-44. 


\section{Figure Legends}

Figure 1. SIRT1 expression in uninvolved cervical epithelium and CIN 1. A. Normal cervical epithelium (H\&E, 100X); B. SIRT1 expression in normal cervical epithelium (100X); C. Abrupt transition from uninvolved cervix epithelium to CIN 1 (H\&E, 100X); D. SIRT1 upregulation in CIN 1 (100X).

Figure 2. SIRT1 expression in CIN 2 AND CIN 3. A. Moderate squamous dysplasia, CIN 2 (H\&E, 100X); B. SIRT1 expression in CIN 2 (100X); C. Severe squamous dysplasia, CIN 3 (H\&E, 100X); D. SIRT1 expression in CIN 3 (100X).

Figure 3. SIRT1 expression in SCC. A. Invasive SCC (H\&E, 40X); B. SIRT1 expression in invasive SCC and in situ SCC (40X); C. Cytoplasmic SIRT1 expression in invasive SCC and in situ SCC (100X); D. Cytoplasmic and nuclear SIRT1 expression in invasive SCC (100X).

Figure 4. p16INK4a overexpression in cervical lesion A. Moderate squamous dysplasia, CIN 2 (H\&E, 40X); B. p16INK4a expression in CIN 2 (40X)

Table 1. Expression of SIRT1 in CIN 1 to 3 and SCC 


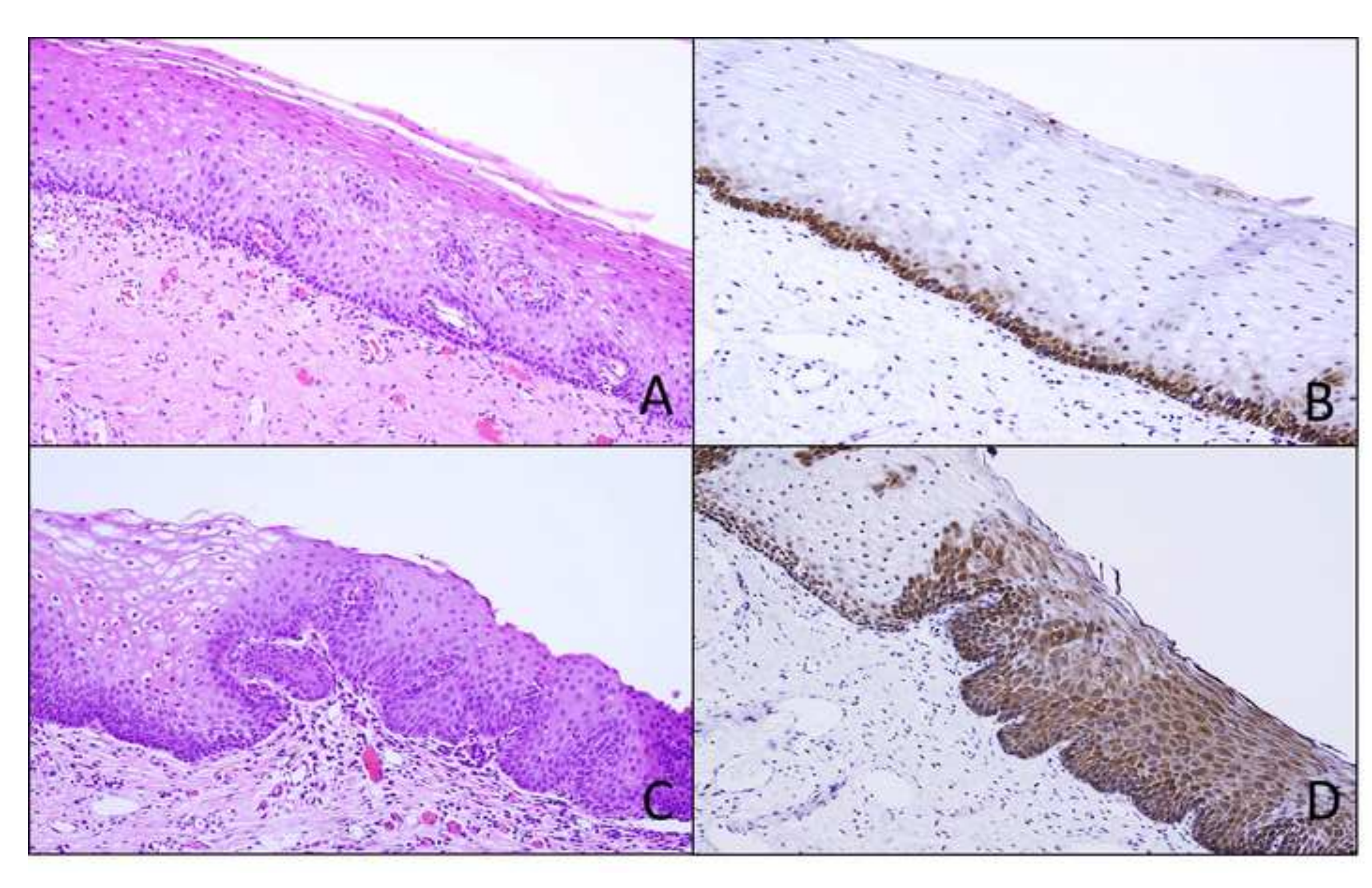

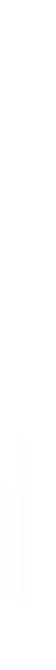

.

.

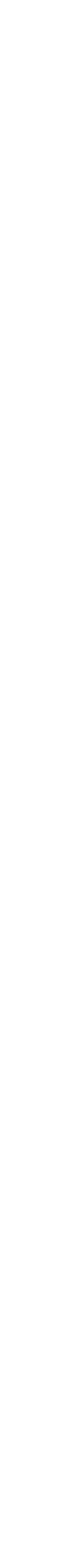




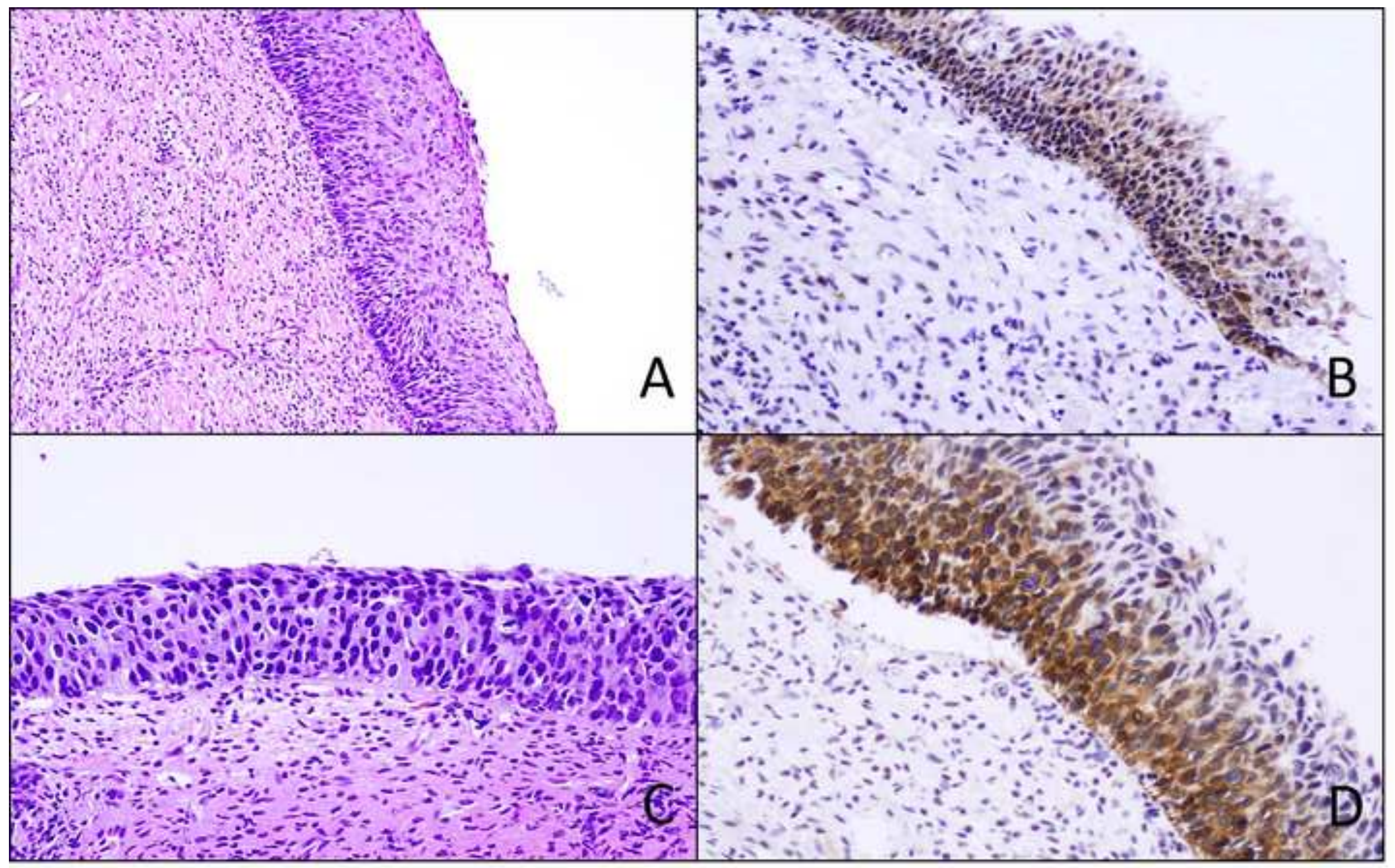




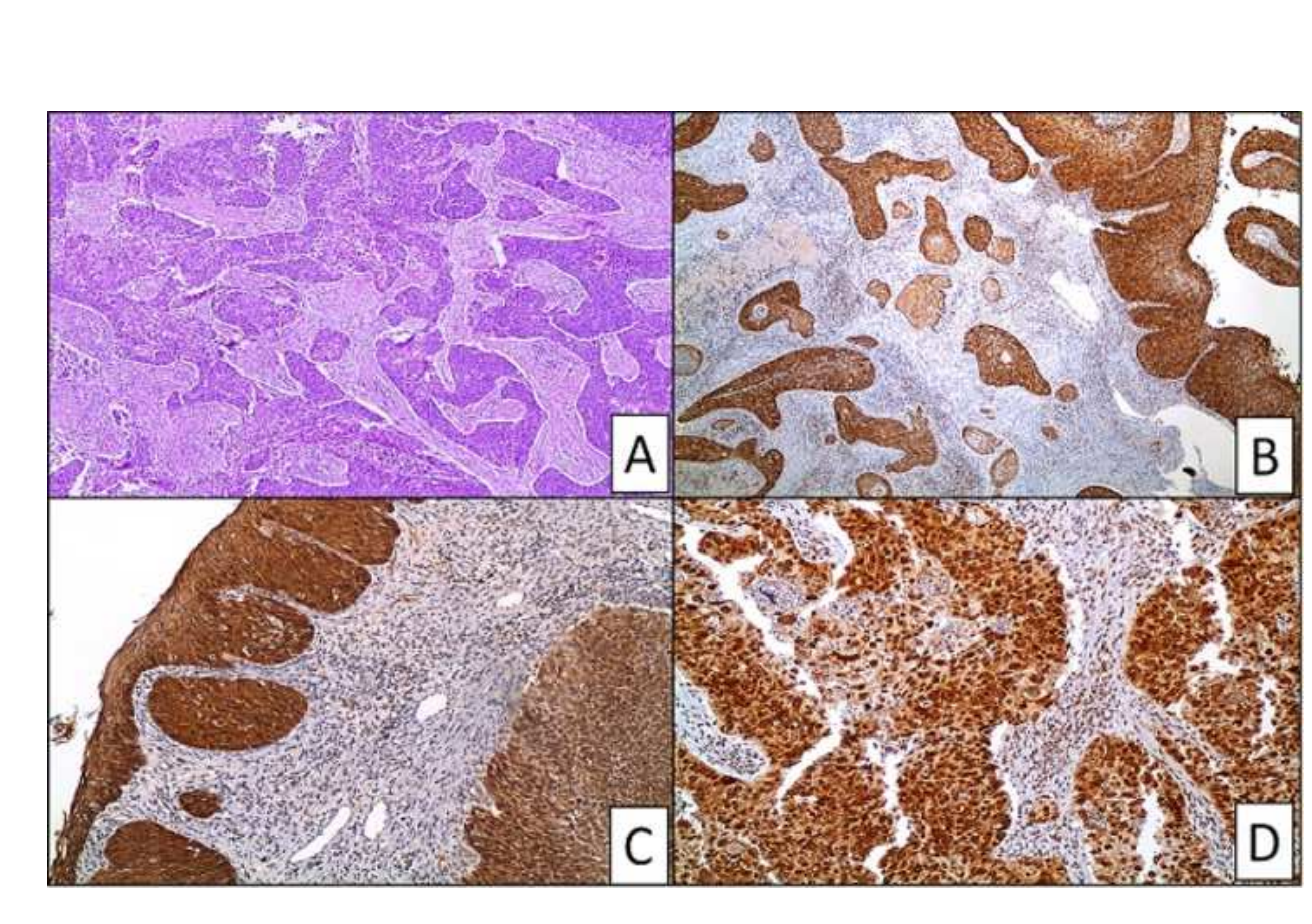

.

.
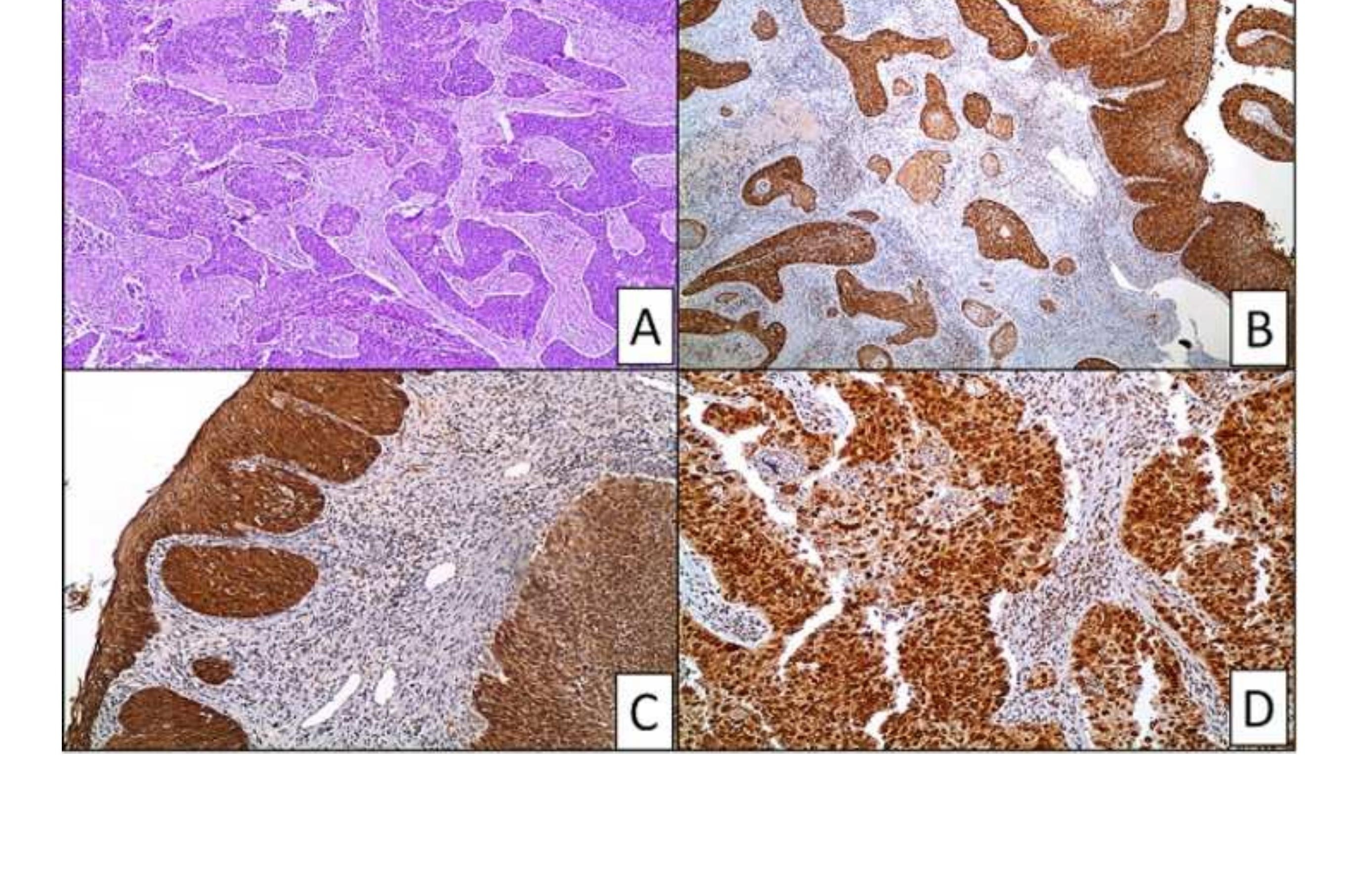
Table 1. Expression of SIRT1 in CIN 1 to 3 and SCC

\begin{tabular}{llllll}
\hline Neoplasm & No. of cases & $\begin{array}{l}\text { Positive } \\
(\text { C only) }\end{array}$ & $\begin{array}{l}\text { Positive } \\
(\text { N only })\end{array}$ & $\begin{array}{l}\text { Positive } \\
(\boldsymbol{N} \& C)\end{array}$ & $\boldsymbol{p}$ value \\
\hline CIN 1 & 29 & $4(13.8 \%)$ & - & - & - \\
CIN 2 & 32 & $13(40.6 \%)$ & - & - & $-{ }^{*}$ \\
CIN 3 & 16 & $8(50 \%)$ & - & - & $0.01^{*}$ \\
mSCC/SCC & 24 & $6(25 \%)$ & $1(4.2 \%)$ & $17(70.8 \%)$ & $0.0001^{\#}$ \\
\hline
\end{tabular}

Abbreviations: cervical intraepithelial neoplasm (CIN); microinvasive squamous cell carcinoma (mSCC); squamous cell carcinoma (SCC); Sirtuin 1 (SIRT1); cytoplasmic stain (C); nuclear stain (N)

${ }^{*} p$ value differences between CIN 1 vs CIN 2/3

${ }^{\#} p$ value differences between CIN $2 / 3$ vs SCC 